\title{
A New Method of Measurement of the True Temperature and Emissivity of Non-Black Body Using One Component of Wien's Spectrum of Thermal Radiation
}

\section{Ya. Svet}

Joint Institute for High Temperatures of the Russian Academy of Sciences, Moscow, Russian Federation Email: vsvetd@mail.ru

Received 11 March 2015; accepted 20 May 2015; published 22 May 2015

Copyright $\odot 2015$ by author and Scientific Research Publishing Inc. This work is licensed under the Creative Commons Attribution International License (CC BY). http://creativecommons.org/licenses/by/4.0/

\section{(c) (i) Open Access}

\begin{abstract}
It is shown that the measurement of only one component of the Wein's spectrum of thermal radiation in range $\lambda T \leq 3000\left({ }^{\circ} \mathrm{C} \mu \mathrm{m}\right)$ is sufficient to estimate the true temperature and spectral emissivity of the selected component with sufficient accuracy, although more than one hundred years this statement was considered as incorrect. The proposed method is based on the formation of the linear dependence of the logarithm of the emissivity of not real wavelengths and artificially generated "virtual" spectral components.
\end{abstract}

Keywords

True Temperature, Spectral Emissivity, Wein's Law

\section{Introduction}

Since the discovery of Wien's exponential law of thermal radiation the concepts of emissivity and black-body factor were firmly established in physics. And measuring of the true temperature and emissivity became a problem "idem per idem" (the same through the same): To measure the true temperature it is necessary to know the emissivity and vice versa. A close look at this problem (on which the author spent more than sixty years) showed that its solution exists. Moreover, it was found that the measurement of any of the spectral components of radiation following to an exponential Wien's law is enough to determine the value of true temperature and spectral emissivity of this component. Below we shall describe the proposed method which implements this 
statement ${ }^{1}$.

\section{Method}

The method is based on the principle of double spectral ratio, based on the linear dependence of the logarithm of the emissivity for three selected components of the spectrum of thermal radiation, proposed in [1] [2], which is widely represented in the literature [3]. However, application of this principle is limited by the requirement on the linearity a priory. This takes place practically only for wavelengths with very closed spacing, which in turn leads to increasing of the value of equivalent wavelength, i.e. to an unacceptable increasing error of measured temperature.

In proposed method a linear dependence of the three components, two of which are "virtual", is generated artificially relative the selected spectral component and the requirement of proximity of wavelengths is eliminated.

Thus, only one spectral component is extracted from the Wien's spectrum that is logarithmic,

$$
\operatorname{Ln} U_{1}=\operatorname{Ln} \varepsilon_{1}-\frac{C_{2}}{\lambda_{1} T}
$$

where:

$\lambda_{1}$-wavelength;

$T$-temperature in ${ }^{\circ} \mathrm{C}$;

$\varepsilon$ - spectral emissivity at wavelength $\lambda_{1}$;

$\mathrm{C}_{2}-14,388 \mu \mathrm{m} /{ }^{\circ} \mathrm{C}$ - second pyrometer constant.

The value of the second pyrometer signal is determining by multiplying $\operatorname{Ln} U_{1}$ on the ratio $\lambda_{1} / \lambda_{2}$ which is proportional to a "virtual" spectral component at a selected wavelength $-\lambda_{2}$, more or less than $\lambda_{1}$. For example, let's choose $\lambda_{2}>\lambda_{1}$

$$
\operatorname{Ln} U_{2}=\frac{\lambda_{1}}{\lambda_{2}} \operatorname{Ln} U_{1}=\frac{\lambda_{1}}{\lambda_{2}} \operatorname{Ln} \varepsilon_{1}-\frac{C_{2}}{\lambda_{2} T}=\operatorname{Ln} \varepsilon_{2}-\frac{C_{2}}{\lambda_{2} T}
$$

where $\operatorname{Ln} \varepsilon_{2}=\frac{\lambda_{1}}{\lambda_{2}} \operatorname{Ln} \varepsilon_{1}$

Further, let's form a third pyrometer signal proportional to the "virtual" component with a wavelength $\lambda_{m}$, which, for example, defined by the half-sum of inverse values of wavelengths

$$
\begin{gathered}
\lambda_{M}=\frac{2 \lambda_{1} \lambda_{2}}{\lambda_{1}+\lambda_{2}}, \text { i.e. } \\
\operatorname{Ln} U_{M}=\frac{\left(\operatorname{Ln} U_{1}+\operatorname{Ln} U_{2}\right)}{2}=\operatorname{Ln} \varepsilon_{M}-\frac{C_{2}}{\lambda_{M} T}
\end{gathered}
$$

where

$$
\operatorname{Ln} \varepsilon_{M}=\frac{\left(\operatorname{Ln} \varepsilon_{1}+\operatorname{Ln} \varepsilon_{2}\right)}{2}=0,5\left(1+\frac{\lambda_{1}}{\lambda_{2}}\right) \operatorname{Ln} \varepsilon_{1}
$$

Thus

$$
\operatorname{Ln} U_{M}=0,5\left(1+\frac{\lambda_{1}}{\lambda_{2}}\right) \operatorname{Ln} \varepsilon_{1}-\frac{C_{2}}{\left(\frac{2 \lambda_{1} \lambda_{2}}{\lambda_{1}+\lambda_{2}}\right) T}
$$

Having these three pyrometer signals $\operatorname{Ln} U_{1}, \operatorname{Ln} U_{m}$ and $\operatorname{Ln} U_{2}$ we can form a linear dependence of the logarithm of the emissivity versus wavelength.

Graphically this is shown in Figure 1, where the emissivity's of these three signals marked by ordinates $\operatorname{Ln} \varepsilon_{1}$, $\operatorname{Ln} \varepsilon_{2}$ and $\operatorname{Ln} \varepsilon_{m}$ and, as it is seen (dotted lines) they form a linear polyline of emissivity versus wave-

${ }^{1}$ Patent is pending, D. Ya. Svet, patent application No. 2014143378 from 28.10.2014. 


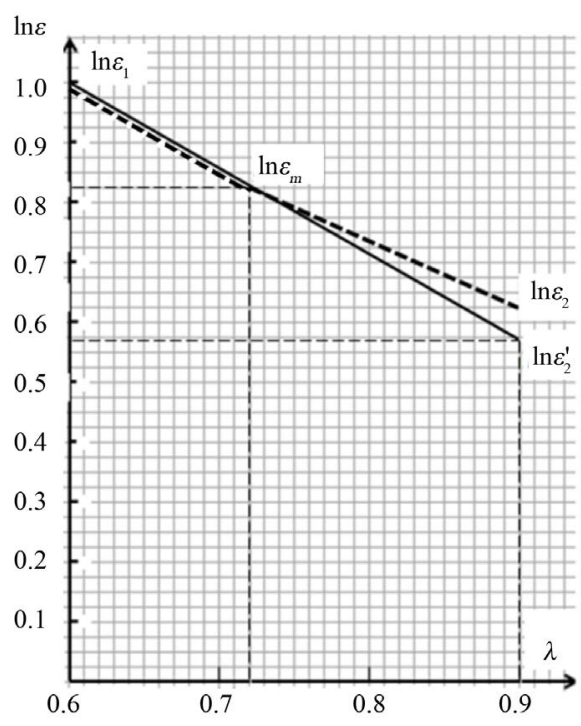

Figure 1. Emissivity versus wavelength.

length.

For the linearization of this dependence and formation of a linear dependence (this is a straight line $\operatorname{Ln} \varepsilon_{1}-\operatorname{Ln} \varepsilon_{m}-\operatorname{Ln} \varepsilon_{2}$ on Figure 1) we shall use expression from [4].

This formula determines the value of the true temperature at known a'priory linearity of logarithm of emissivity from the wavelength and has the form:

$$
\begin{aligned}
V & =K\left\{\left[\frac{\lambda_{M}}{\lambda_{M}-\lambda_{2}}\right] \operatorname{Ln} U_{2}-\left[\frac{\lambda_{M}}{\lambda_{M}-\lambda_{1}}\right] \operatorname{Ln} U_{1}-\left[\frac{\lambda_{M} \lambda_{1}-\lambda_{M} \lambda_{2}}{\left(\lambda_{M} \lambda_{2}-\lambda_{1} \lambda_{2}-\lambda_{M}^{2}+\lambda_{1} \lambda_{M}\right)}\right] \operatorname{Ln} U_{M}\right\} \\
& =K\left\{\left[\frac{\lambda_{M}}{\lambda_{M}-\lambda_{2}}\right]\left(\operatorname{Ln} \varepsilon_{2}-\frac{C_{2}}{\lambda_{2} T}\right)-\left[\frac{\lambda_{M}}{\lambda_{M}-\lambda_{1}}\right]\left(\operatorname{Ln} \varepsilon_{1}-\frac{C_{2}}{\lambda_{1} T}\right)-\left[\frac{\lambda_{M} \lambda_{1}-\lambda_{M} \lambda_{2}}{\lambda_{M} \lambda_{2}-\lambda_{1} \lambda_{2}-\lambda_{M}^{2}+\lambda_{1} \lambda_{M}}\right]\left(\operatorname{Ln} \varepsilon_{M}-\frac{C_{2}}{\lambda_{M} T}\right)\right\},
\end{aligned}
$$

where $V$ determines the true temperature at the wavelength $\Lambda=\lambda_{1} \lambda_{2} /\left(\lambda_{2}-\lambda_{1}\right)$, at the linear dependence of the logarithm of the emissivity from the wavelength, and $K=$ const which is set during calibration.

Usually for method of double spectral ratio the relation $\operatorname{Ln} U_{1} U_{2} / U_{M}^{2}$ is using and that requires equidistant spacing of wavelengths; wherein the equivalent wavelength is rather big that increase the error of measuring. According [4] spacing of wavelengths can be arbitrary and not equidistant and that can decrease equivalent wavelength and improve the accuracy of measured temperature.

Thus, substituting the values of $\ln \varepsilon_{1}, \ln \varepsilon_{2}$ and $\ln \varepsilon_{m}$ in Equation (6) we will receive

$$
\begin{aligned}
V & =\left(\frac{\lambda_{M}}{\lambda_{M}-\lambda_{2}}\right) \operatorname{Ln} \varepsilon_{2}-\left(\frac{\lambda_{M}}{\lambda_{M}-\lambda_{1}}\right) \operatorname{Ln} \varepsilon_{1}-\left[\frac{\lambda_{M} \lambda_{1}-\lambda_{2} \lambda_{M}}{\lambda_{2} \lambda_{M}-\lambda_{1} \lambda_{2}-\lambda_{M}^{2}+\lambda_{1} \lambda_{M}}\right] \operatorname{Ln} \varepsilon_{M}-\frac{C_{2}}{\Lambda T} \\
& =\left\{\left(\frac{\lambda_{1}}{\lambda_{2}}\right)\left(\frac{\lambda_{M}}{\lambda_{M}-\lambda_{2}}\right) \operatorname{Ln} \varepsilon_{1}-\left(\frac{\lambda_{M}}{\lambda_{M}-\lambda_{1}}\right) \operatorname{Ln} \varepsilon_{1}-\left[\frac{\lambda_{1} \lambda_{M}-\lambda_{2} \lambda_{M}}{\lambda_{2} \lambda_{M}-\lambda_{1} \lambda_{2}-\lambda_{M}^{2}+\lambda_{1} \lambda_{M}}\right]\left(\frac{1+\frac{\lambda}{\lambda}}{2}\right) \operatorname{Ln} \varepsilon_{1}\right\}-\frac{C_{2}}{\Lambda T},
\end{aligned}
$$

And get the expression for the "resultant" emissivity in the form of $\operatorname{Ln} E$

$$
\operatorname{Ln} E=\left\{\frac{\lambda_{1}}{\lambda_{2}}\left[\frac{\lambda_{M}}{\lambda_{M}-\lambda_{2}}\right]-\left[\frac{\lambda_{M}}{\lambda_{M}-\lambda_{1}}\right]-\left[\frac{\lambda_{1} \lambda_{M}-\lambda_{2} \lambda_{M}}{\lambda_{2} \lambda_{M}-\lambda_{1} \lambda_{2}-\lambda_{M}^{2}+\lambda_{1} \lambda_{M}}\right]\left(\frac{1+\frac{\lambda_{1}}{\lambda_{2}}}{2}\right)\right\} \operatorname{Ln} \varepsilon_{1}
$$


If to follow to the linear dependence of emissivity from wavelength the "resultant" emissivity must be equal to zero, $\operatorname{Ln} E=0$. However, it is obvious that $\operatorname{Ln} E \neq 0$.

This can be seen from the formula (7) for $\mathrm{V}$ and illustrated in Figure 1, where: $\operatorname{Ln} E=f(\lambda)$ is not straight line and linear polyline marked by dots. Therefore for linearization of this relationship it is necessary to change the values $\operatorname{Ln} \varepsilon_{2}$ or $\operatorname{Ln} \varepsilon_{m}$.

Let's change the value of $\operatorname{Ln} \varepsilon_{2}$. From Equation (7) it is seen that the values $\operatorname{Ln} \varepsilon_{1}, \operatorname{Ln} \varepsilon_{2}$ and $\operatorname{Ln} \varepsilon_{m}$ differ only by constant coefficients.

Thus, we determine the value of $\operatorname{Ln} \varepsilon_{2}^{*}$, where the "resultant" dependence of logarithm of emissivity will be linear.

$$
\operatorname{Ln} \varepsilon_{2}^{*}=\frac{\left\{\left(-\frac{\lambda_{M}}{\lambda_{M}-\lambda_{1}}\right)-\left[\frac{\left(\lambda_{1} \lambda_{M}-\lambda_{2} \lambda_{M}\right)}{\left(\lambda_{2} \lambda_{M}-\lambda_{1} \lambda_{2}-\lambda_{M}^{2}+\lambda_{1} \lambda_{M}\right)}\right]\left(\frac{1+\frac{\lambda_{1}}{\lambda_{2}}}{2}\right)\right\}}{\left(\frac{\lambda_{M}}{\lambda_{M}-\lambda_{2}}\right)}
$$

Substituting the expression for $\operatorname{Ln} \varepsilon_{2}^{*}$ into $\operatorname{Ln} U_{2}$, we obtain the value $\operatorname{Ln} U_{2}^{*}$ as:

$$
\begin{gathered}
\operatorname{Ln} U_{2}^{*}=\operatorname{Ln} U_{2}-\Delta \operatorname{Ln} U_{2} \\
\text { where } \Delta \operatorname{Ln} U_{2}=\operatorname{Ln} U_{2}-\operatorname{Ln} U_{2}^{*}=\operatorname{Ln} \varepsilon_{1}-\operatorname{Ln} \varepsilon_{2}^{*}
\end{gathered}
$$

Thus, the expression (6) for $V$, specifying the desired value of the true temperature at a linear dependence of the logarithm of the emissivity will have the form:

$$
\begin{gathered}
V^{*}=\left(\frac{\lambda_{M}}{\lambda_{M}-\lambda_{2}}\right) \operatorname{Ln} U_{2}^{*}-\left(\frac{\lambda_{M}}{\lambda_{M}-\lambda}\right) \operatorname{Ln} U_{1}-\left[\frac{\left(\lambda_{1} \lambda_{M}-\lambda_{2} \lambda_{M}\right)}{\left(\lambda_{2} \lambda_{M}-\lambda_{1} \lambda_{2}-\lambda_{M}^{2}+\lambda_{1} \lambda_{M}\right)}\right] \operatorname{Ln} U_{M} \\
\text { or } V^{*}=\operatorname{Ln} E^{*}-\frac{C_{2}}{\Lambda T}
\end{gathered}
$$

Moreover,

$$
\operatorname{Ln} E^{*}=\left[\frac{\lambda_{M}}{\left(\lambda_{M}-\lambda_{2}\right)}\right] \operatorname{Ln} \varepsilon_{2}^{*}-\left[\frac{\lambda_{M}}{\left(\lambda_{M}-\lambda_{1}\right)}\right] \operatorname{Ln} \varepsilon_{1}-\left[\frac{\left(\lambda_{1} \lambda_{M}-\lambda_{2} \lambda_{M}\right)}{\left(\lambda_{2} \lambda_{M}-\lambda_{1} \lambda_{2}-\lambda_{M}^{2}+\lambda_{1} \lambda_{M}\right)}\right] \operatorname{Ln} \varepsilon_{M}=0
$$

Thus, the desired value of the true temperature will be equal to

$$
C_{2}=\frac{T}{V \Lambda}
$$

where,

$$
\Lambda=\frac{\lambda_{1} \lambda_{2}}{\lambda_{2}-\lambda_{1}}
$$

And the emissivity will be equal to

$$
\operatorname{Ln} \varepsilon_{1}=\operatorname{Ln} U_{1}+\frac{C_{2}}{\lambda_{1} T}
$$

\section{Example}

As an example of proposed method let's present the result of calculation of the true temperature for steel; data are taken from [5]. This work contains the following data:

Steel $\mathrm{Fe}+0.53 \% \mathrm{C} ; T=1600^{\circ} \mathrm{C}$ at a wavelength $\lambda_{1}=0.6 \mu \mathrm{m}, \varepsilon_{1}=0.39 ; \operatorname{Ln} \varepsilon_{1}=-0.95$, the value of 
$\operatorname{Ln} U_{1}=\operatorname{Ln} \varepsilon_{1}-C_{2} / \lambda_{1} T=-0.95-\left(\frac{14,388}{0.6} \times 1600\right)=-15.937$

Let's now choose a second "virtual" wavelength from the spectrum of thermal radiation, for example, $\lambda_{2}=0.9 \mu \mathrm{m}$. Then the second pyrometer signal can be written as:

$$
\operatorname{Ln} U_{2}=\lambda_{1} / \lambda_{2} \operatorname{Ln} U_{1}=\lambda_{1} / \lambda_{2} \operatorname{Ln} \varepsilon_{1}-C_{2} / \lambda_{2} T=\operatorname{Ln} \varepsilon_{2}-C_{2} / \lambda_{2} T \text {, where } \lambda_{1} / \lambda_{2}=0.6 / 0.9=0.666 \text {. }
$$

Then $\operatorname{Ln} \varepsilon_{2}=\lambda_{1} / \lambda_{2} \operatorname{Ln} \varepsilon_{1}=0.666 \operatorname{Ln} \varepsilon_{1}$, and $\operatorname{Ln} U_{2}=0.666 \operatorname{Ln} \varepsilon_{1}-C_{2} / 0.9 T=-10.625$.

The third signal $\operatorname{Ln} U_{m}$ which is equal to half the sum of the first two will be equal to

$$
\operatorname{Ln} U_{m}=\left(\operatorname{Ln} U_{1}+\operatorname{Ln} U_{2}\right) / 2=\operatorname{Ln} \varepsilon_{m}-C_{2} / \lambda_{m} T=(-15.9375-10.625) / 2=-13.28
$$

where: $\operatorname{Ln} \varepsilon_{m}=0.5\left(\operatorname{Ln} \varepsilon_{1}+\operatorname{Ln} \varepsilon_{2}\right)$, and $\operatorname{Ln} \varepsilon_{m}=0.5\left(1+\lambda_{1} / \lambda_{2}\right) \operatorname{Ln} \varepsilon_{1}=0.5(1+0.66) \operatorname{Ln} \varepsilon_{1}=0.833 \operatorname{Ln} \varepsilon_{1}$, and

$$
\lambda_{m}=2 \lambda_{1} \lambda_{2} /\left(\lambda_{2}+\lambda_{1}\right)=0.72
$$

To provide the linearity of logarithm of emissivity let's change the value $\operatorname{Ln} \varepsilon_{2}$, using formula (9), and

$$
\operatorname{Ln} \varepsilon_{2}^{*}=(-10+6 \times 0.833) / 4=0.5833
$$

Now by substituting $\operatorname{Ln} \varepsilon_{2}^{*}$ into the expression for $\operatorname{Ln} U_{2}$, we will get the value of $\operatorname{Ln} U_{2}^{*}$ in the form of

$$
\operatorname{Ln} U_{2}^{*}=\operatorname{Ln} U_{2}-\Delta \operatorname{Ln} U_{2} \text {, }
$$

where: $\Delta \operatorname{Ln} U_{2}=\operatorname{Ln} U_{2}-\operatorname{Ln} U_{2}^{*}=\operatorname{Ln} \varepsilon_{2}-\operatorname{Ln} \varepsilon_{2}^{*}=0.666-0.583=0.083$

Therefore $\operatorname{Ln}_{2}^{*}=-10.625-0.0833=10.541$ and the value of $V^{*}$ in accordance with formula (11) will be equal to

$$
\begin{gathered}
V^{*}=-4 \times 10.5416-6 \times 15.9375+10 \times 13.2812-\frac{14,388}{1.8 T}=-42.1666-95.625+132.8125-\frac{14,388}{1.8 T}=-4.9796 \\
\text { or }-4.97966=4 \operatorname{Ln} \varepsilon_{2}^{*}-6 \operatorname{Ln} \varepsilon_{1}+10 \times 0.833 \cdots 3-\frac{14,388}{1.8 T}=-4.9796 .
\end{gathered}
$$

Therefore, the required temperature will be equal to $T=\frac{14,388}{1.8} * 4.97966=1605.19^{\circ} \mathrm{C}$

And the resulting error will be

$$
\delta T=\frac{|1605.19-1600|}{1600} * 100 \%=0.4 \%
$$

The value of the logarithm of the emissivity will be equal to

$$
\operatorname{Ln} \varepsilon_{1}=\operatorname{Ln} U_{1}-C_{2} / \lambda_{1} T=15.9375-\frac{14,388}{0.6} \times 1605.19=0.998 .
$$

And the resulting error will be

$$
\delta \varepsilon=\frac{|0.998-0.95|}{0.95} * 100 \%=5 \% \text { at a wavelength of } \lambda_{1}=0.6 \mu \mathrm{m} .
$$

From this example we can see that proposed method based on selection of "virtual" components provides rather good accuracy.

Note that if to select the second "virtual" component of the spectrum with a wavelength $\lambda_{2}<\lambda_{1}$, that will reduce the equivalent wavelength $\Lambda$, and the error of true temperature will be less. For example for $\lambda_{2} / \lambda_{1}=1.5$, i.e. $\lambda_{2}=0.4 \mu \mathrm{m}$, then $\Lambda=\lambda_{1} \lambda_{2} /\left(\lambda_{2}-\lambda_{1}\right)=1.2$ and the error will be reduced in 1.5 times, i.e.

$$
\delta T=0.25^{\circ} \mathrm{C} \text {. }
$$

\section{Conclusion}

The described method and calculations performed on its base show that it can estimate fairly accurately the value of the true temperature and emissivity using only one real spectral component and two complementary "virtual" 
components of the emissivity spectrum which follows to Wien's law and additional virtual spectral components can be selected rather arbitrarily. From a mathematical point of view, the proposed method is not rigorous, because formally Equation (1) includes two unknown parameters and the proposed heuristic approach is rather geometric in nature, but it provides, and quite simply, accurate measurements of true temperature and emissivity in spectral region $\lambda T \leq 3000 \mu \mathrm{m}^{\circ} \mathrm{C}$.

\section{Acknowledgements}

I am grateful to the staff of the Academic Council of the Joint Institute for High Temperatures of the Russian Academy of Sciences for fruitful discussion of this work.

\section{References}

[1] Svet, D.Ya. (1975) Color Pyrometer of True Temperature. Certificate of Invention, No. 476464. Bulletin of Inventions, Moscow.

[2] Svet, D.Ya. (1982) Optical Methods for Measuring the True Temperature. Nauka, Moscow, 145.

[3] Hornbeck, G.A. (1962) Temperature: Its Measurement and Control in Science and Industry. Reinhold V.I., New-York, 262.

[4] Svet, D.Ya. (1985) 3-Wavelength Radiation Pyrometer. Certificate of Invention No. 1284336. Bulletin of Inventions, Moscow.

[5] Shvarev, K.M., Gushchin, V.S., Baum, B.A. and Held, V.P. (1979) Optical Constants of Iron-Carbon Alloys in the Temperature Range $20^{\circ} \mathrm{C}-1600^{\circ} \mathrm{C}$. Teplofizika, 17, 66-71. 